\title{
REMARK ON THE GENERALIZED PUTNAM-FUGLEDE THEOREM
}

\author{
TAKASHI YOSHINO
}

AbStract. If $A^{*}$ is $M$-hyponormal, $B$ is dominant, and if $C A=B C$, then $C A^{*}=$ $B^{*} C$.

Let $\mathscr{H}$ be a Hilbert space. An operator $A \in \mathscr{B}(\mathscr{H})$ is dominant if $\operatorname{range}(A-\lambda I)$ $\subset \operatorname{range}(A-\lambda I)^{*}$ for all $\lambda \in \sigma(A)$, or, equivalently, for all $\lambda \in \mathbf{C}$ because

$$
\operatorname{range}(A-\lambda I)=\operatorname{range}(A-\lambda I)^{*}=\mathscr{H}
$$

for $\lambda \notin \sigma(A)$. By the result of Douglas [1], it is equivalent to the existence of a real number $M_{\lambda}$ for each $\lambda \in \mathrm{C}$ such that

$$
\left\|(A-\lambda I)^{*} x\right\| \leqslant M_{\lambda}\|(A-\lambda I) x\|
$$

for all $x \in \mathscr{H}$. If there is a constant $M$ such that $M_{\lambda} \leqslant M$ for all $\lambda \in \mathbf{C}, A$ is called $M$-hyponormal, and if $M=1, A$ is hyponormal.

Stampfli and Wadhwa [5] showed that if $A^{*} \in \mathscr{B}(\mathscr{H})$ is hyponormal, $B \in \mathscr{B}(\mathscr{K})$ is dominant, $C \in \mathscr{B}(\mathscr{H}, \mathscr{K})$ is injective and has dense range, and if $C A=B C$, then $A$ and $B$ are normal.

Radjabalipour [3] improved this result by allowing $A^{*}$ to be $M$-hyponormal. Of course, the condition that $A$ and $B$ are normal allows one to conclude immediately by the usual Putnam-Fuglede theorem that $C A^{*}=B^{*} C$.

Moore, Rogers and Trent [2] showed that, if $A^{*}$ and $B$ are both $M$-hyponormal, the conclusion $C A^{*}=B^{*} C$ can be reached with no restrictions on $C$ at all.

We show that this result can also be improved by allowing $B$ to be dominant.

We need the following two lemmas:

LemMa 1 [4]. Let $A$ be dominant and let $\mathscr{M}$ be an invariant subspace of $A$ for which $A \mid \mathscr{M}$ is normal. Then $\mathscr{M}$ reduces $A$.

Lemma 2. The restriction $A \mid \mathscr{M}$ of the dominant (respectively, M-hyponormal) operator $A$ on $\mathscr{H}$ to an invariant subspace $\mathscr{M}$ of $A$ is dominant (respectively, M-hyponormal).

PROOF. Let $P_{\mathscr{M}}$ be the orthogonal projection from $\mathscr{H}$ to $\mathscr{M}$. Then

$$
\begin{aligned}
\left\|(A \mid \mathscr{M}-\lambda I)^{*} x\right\| & =\left\|P_{\mathscr{M}}(A-\lambda I)^{*} x\right\| \leqslant\left\|(A-\lambda I)^{*} x\right\| \\
& \leqslant M_{\lambda}(\operatorname{resp} . M)\|(A-\lambda I) x\|=M_{\lambda}(\operatorname{resp} . M)\|(A \mid \mathscr{M}-\lambda I) x\|
\end{aligned}
$$

for each $\lambda \in \mathbf{C}$ and all $x \in \mathscr{M}$.

Received by the editors November 26, 1984.

1980 Mathematics Subject Classification. Primary 47B20; Secondary 47B99.

(c1985 American Mathematical Society $0002-9939 / 85 \$ 1.00+\$ .25$ per page 
TheOREM. If $A^{*} \in \mathscr{B}(\mathscr{H})$ is $M$-hyponormal, $B \in \mathscr{B}(\mathscr{K})$ is dominant, and if $C A=B C$ for $C \in \mathscr{B}(\mathscr{H}, \mathscr{K})$, then $C A^{*}=B^{*} C$.

Proof. From $C A=B C$ we know that $(\operatorname{ker} C)^{\perp}$ and $(\text { range } C)^{-}$are invariant subspaces of $A^{*}$ and $B$, respectively. Hence $A^{*} \mid(\operatorname{ker} C)^{\perp}$ is $M$-hyponormal and $B \mid(\text { range } C)^{-}$is also dominant by Lemma 2 . By the decompositions $\mathscr{H}=(\operatorname{ker} C)^{\perp}$ $\oplus(\operatorname{ker} C)$ and $\mathscr{K}=(\text { range } C)^{-} \oplus\left\{(\text { range } C)^{-}\right\}^{\perp}$, we have

$$
A=\left(\begin{array}{cc}
A_{1} & 0 \\
* & A_{2}
\end{array}\right), \quad B=\left(\begin{array}{cc}
B_{1} & * \\
0 & B_{2}
\end{array}\right), \quad C=\left(\begin{array}{cc}
C_{1} & 0 \\
0 & 0
\end{array}\right) .
$$

Here $A_{1}^{*}=A^{*} \mid(\operatorname{ker} C)^{\perp}$ is $M$-hyponormal, $B_{1}=B \mid(\text { range } C)^{-}$is dominant and $C_{1}$ is injective with dense range. We obtain $C_{1} A_{1}=B_{1} C_{1}$ from $C A=B C$. Hence, $A_{1}$ and $B_{1}$ are normal by [3, Theorem 3(a)] and $C_{1} A_{1}^{*}=B_{1}^{*} C_{1}$ by the usual Putnam-Fuglede theorem. Then, by Lemma $1,(\operatorname{ker} C)^{\perp}$ and $(\operatorname{range} C)^{-}$reduces $A^{*}$ and $B$ to normal operators, respectively. Therefore, we have

$$
A=\left(\begin{array}{cc}
A_{1} & 0 \\
0 & A_{2}
\end{array}\right), \quad B=\left(\begin{array}{cc}
B_{1} & 0 \\
0 & B_{2}
\end{array}\right) .
$$

Hence we obtain $C A^{*}=B^{*} C$.

\section{REFERENCES}

1. R. G. Douglas, On majorization, factorization, and range inclusion of operators on Hilbert space, Proc. Amer. Math. Soc. 17 (1966), 413-415.

2. R. L. Moore, D. D. Rogers and T. T. Trent, A note on intertwining M-hyponormal operators, Proc. Amer. Math. Soc. 83 (1981), 514-516.

3. M. Radjabalipour, On majorization and normality of operators, Proc. Amer. Math. Soc. 62 (1977). 105-110.

4. J. G. Stampfli and B. L. Wadhwa, An asymmetric Putnam-Fuglede theorem for dominant operators, Indiana Univ. Math. J. 25 (1976), 359-365.

5. , On dominant operators, Monatsh. Math. 84 (1977), 143-153.

Department of Mathematics, College of General Education, TÓhoku University, Kawauchi, SENDAI, JAPAN 